\title{
IFP NEAR-RINGS
}

\author{
D. RAMAKOTAIAH and G. KOTESWARA RAO
}

(Received 21 February; revised 3 August 1978)

Communicated by R. Lidl

\begin{abstract}
The set of all nilpotent elements in an IFP near-ring is characterized and necessary and sufficient conditions for the set of all nilpotent elements of an IFP near-ring to form an ideal are obtained.
\end{abstract}

Subject classification (Amer. Math. Soc.(MOS) 1970): 16 A 76.

\section{Introduction}

IFP near-rings are introduced in Bell $(1970,1971)$, Ligh $(1970,1972)$ and Pilz (1977). Commutative near-rings, near-rings without nilpotent elements, right duo-near-rings are some examples of IFP near-rings. In this paper we wish to obtain necessary and sufficient conditions for the set of all nilpotent elements of an IFP near-ring to form an ideal. This paper is divided into three sections. In Section 1 we present preliminaries. In Section 2 we characterize the set of all nilpotent elements in an IFP near-ring. In Section 3 different types of prime ideals are introduced and necessary and sufficient conditions for the set of all nilpotent elements of an IFP near-ring to form an ideal are obtained.

An algebraic system $N=(N,+, ., 0)$ is called a near-ring if and only if (i) $(N,+, 0)$ is a group, (ii) $(N,$.$) is a semi-group, (iii) a(b+c)=a b+a c$ for all $a, b, c$ in $N$ and (iv) $0 a=0$ for all $a$ in $N$. A subset $K$ of $N$ is said to be an ideal of $N$ if (i) $(K,+)$ is a normal subgroup of $(N,+)$, (ii) $(a+k) b-a b$ belongs to $K$ for all $a, b$ in $N$ and $k \in K$, and (iii) $a k \in K$ for all $a \in N$ and $k \in K$. If (i) and (ii) are satisfied 
then $K$ is called a right ideal of $N$. An element $a$ in $N$ is said to be nilpotent if $a^{n}=0$ for some positive integer $n$. A near-ring $N$ is said to fulfil the insertion-offactors-property (IFP) provided that for all $a, b, n \in N: a b=0$ implies $a n b=0$.

If $M$ is any subset of a near-ring $N$, then $A(M)$ stands for the set $\{a \in N: M a=0\}$.

\section{2}

Throughout this section $N$ stands for an IFP near-ring. If $K$ is a subset of $N$, $P(K)$ stands for $\bigcup_{k \in K} A(k)$ and $N^{*}$ stands for the set of all non-zero elements of $N$.

LEMMA 2.1. If $n_{1}, n_{2} \in N$, then $A\left(n_{1} n_{2}\right) \supseteq A\left(n_{1}\right) \cup A\left(n_{2}\right)$.

The proof is easy and will be omitted.

If $K$ is a sub-semi-group of $(N,$.$) we shall denote the complement of K \cup P(K)$ by $L(K)$.

THEOREM 2.2. Let $K$ be a maximal sub-semi-group in $N^{*}$. Then for each $y \in L(K)$, there exists an $x \in K$ such that $x y$ is nilpotent.

Proof. Let $y \in L(K)$ and $M$ be the sub-semi-group of $(N$, .) generated by $K \cup\{y\}$. Since $y \notin K, M$ contains $K$ properly. Therefore, $0 \in M$. Now there exist $x_{1}, x_{2}, \ldots, x_{n}$ in $K$ such that $x_{1} y^{i_{1}} x_{2} y^{i_{2}} \ldots x_{n} y^{i_{n}}=0$. Put $x=x_{1} x_{2} \ldots x_{n}$. Clearly $x \in K$. Since $N$ is an IFP near-ring, inserting $x_{1}, x_{2}, \ldots, x_{n}$ suitably in $x_{1} y^{i_{1}} x_{2} y^{i_{2}} \ldots x_{n} y^{i_{n}}=0$, we obtain $(x y)^{i_{1}+i_{2}+\ldots+i_{n}}=0$. Therefore $x y$ is nilpotent.

Definition 2.3. If $N$ has no multiplicative sub-semi-group in $N^{*}$, put $Q(N)=N$. If $N$ has multiplicative sub-semi-group in $N^{*}$, define $Q(N)=N \backslash \bigcup_{\alpha \in \Delta} K_{\alpha}$ where $\left\{K_{\alpha}\right\}_{\alpha \in \Delta}$ is the family of all maximal sub-semi-groups in $N^{*}$.

THEOREM 2.4. $Q(N)$ is precisely the set of all nilpotent elements of $N$.

Proof. Suppose $N$ has no sub-semi-group in $N^{*}$. Then each element of $N$ is nilpotent. In this case $Q(N)$ is precisely the set of all nilpotent elements of $N$. Assume that $N$ has sub-semi-groups in $N^{*}$. Let $\left\{K_{\alpha}\right\}_{\alpha \in \Delta}$ be the family of maximal sub-semi-groups in $N^{*}$. Since each $K_{\alpha}$ contains no nilpotent elements, $Q(N)$ contains all the nilpotent elements of $N$. Suppose $x \in Q(N)$ is not nilpotent. Then $K=\left\{x, x^{2}, \ldots\right\}$ is a multiplicative sub-semi-group in $N^{*}$ and it can be extended to a maximal sub-semi-group $K^{\prime}$ in $N^{*}$. Since $x \in K^{\prime}, x \notin Q(N)$, a contradiction. Therefore $Q(N)$ is precisely the set of all nilpotent elements of $N$. 
In this section we introduce three types of prime ideals and the radicals associated with these prime ideals. We obtain necessary and sufficient conditions for the set of all nilpotent elements of an IFP near-ring to form an ideal.

Definition 3.1. An ideal $P$ of a near-ring $N$ is a prime ideal of type 2 if for all $x, y \in N, x y \in P$ implies $x \in P$ or $y \in P$.

Definition 3.2. An ideal $P$ of a near-ring $N$ is a prime ideal of type 1 if for all $x, y \in N, x N y \subseteq P$ implies $x \in P$ or $y \in P$.

DefintTION 3.3. An ideal $P$ of a near-ring $N$ is a prime ideal of type 0 if for all finite sets $\alpha_{1}, \alpha_{2}, \ldots, \alpha_{n}$ of ideals of $N, \alpha_{1} \alpha_{2} \ldots \alpha_{n} \subseteq P$ implies at least one $\alpha_{i} \subseteq P$.

A prime ideal of type 0 is a prime ideal in the sense of Ramakotaiah (1967). In the case of commutative rings all the above definitions are equivalent whereas in the case of non-commutative rings the last two definitions are equivalent. However, in the case of near-rings the above three definitions are not equivalent. It is easy to check that a prime ideal of type 2 is a prime ideal of type 1 and a prime ideal of type 1 is a prime ideal of type 0 . We now introduce radicals associated with the three types of prime ideals introduced above.

Definition 3.4. Let $N$ be an arbitrary near-ring. If $N$ has no prime ideals of type $v$, the $v$-prime radical of $N$ is $N$ for $v=0,1,2$. If $N$ has a prime ideal of type $v$, the $v$-prime radical of $N$ is the intersection of all prime ideals of type $v$ for $v=0,1,2$, and hence an ideal.

We denote the $v$-prime radical of $N$ by $P_{v}(N)$ for $v=0,1,2$. The radical $P_{0}(N)$ is the radical $J_{-2}(N)$ is the sense of Ramakotaiah (1967) and some of its properties are investigated in Ramakotaiah (1967). It is clear that $P_{2}(N)$ contains $P_{1}(N)$ and $P_{1}(N)$ contains $P_{0}(N)$. In Ramakotaiah (1967) it is shown that each element of $J_{-2}(N)=P_{0}(N)$ is nilpotent.

If $N$ is an IFP near-ring, we have shown that $Q(N)$ is precisely the set of all nilpotent elements of $N$ (Theorem 2.4), although $Q(N)$ is not an ideal in general. In order that $Q(N)$ be an ideal, some additional conditions must be imposed on $N$. We know that prime ideals of type 1 and type 0 are the same in the case of noncommutative rings. We shall examine the consequences when every prime ideal of type 0 is a prime ideal of type 1 .

THEOREM 3.5. Every prime ideal of type 1 in an IFP near-ring contains all nilpotent elements. 
ProOF. Let $P$ be a prime ideal of type 1 and $a$, a nilpotent element with order of nilpotency $k$. Suppose $a \notin P$. Write $a_{0}=a$. Since $a_{0} \notin P$, there exists $t_{0} \in N$ such that $a_{1}=a_{0} t_{0} a_{0} \notin P$; otherwise $a_{0} \in P$ since $P$ is a prime ideal of type 1 . If we continue this process we obtain two sequences $\left\{a_{n}\right\},\left\{t_{n}\right\}$ such that $a_{n+1}=a_{n} t_{n} a_{n}$, $n=0,1,2, \ldots$ and $a_{n+1} \notin P$. So each $a_{n} \neq 0$. The number of $a$ 's that occur in $a_{k}$ is $2^{k}$ and $a^{2^{k}}=0$. Since $N$ is an IFP near-ring, inserting $t_{0}, t_{1}, \ldots, t_{n-1}$ suitably, it can be shown that $a_{n}=0$, for $n \geqslant k$, a contradiction. Therefore $a \in P$.

COROLLARY 3.6. If $N$ is an IFP near-ring, then $P_{1}(N)$ contains $Q(N)$.

PrOOF. Since every prime ideal of type 1 contains $Q(N), P_{1}(N)$ contains $Q(N)$. In Ramakotaiah (1967), it is shown that each element of $P_{0}(N)$ is nilpotent. Therefore $P_{0}(N)$ is contained in $Q(N)$, and so we have a proof of:

THEOREM 3.7. Let $N$ be an IFP near-ring. If every prime ideal of type 0 is a prime ideal of type 1 , then $Q(N)$ is an ideal.

COROLLARY 3.8. If $N$ is an IFP near-ring which is a ring, then $Q(N)$ is an ideal.

Proof. Since every prime ideal of type 0 is a prime ideal of type $1, Q(N)$ is an ideal of $N$.

THEOREM 3.9. Let $N$ be an IFP near-ring in which each prime ideal of type 0 is a prime ideal of type 1 . Then $P_{0}(N)=J_{-1}(N)$ where $J_{-1}(N)$ (Ramakotaiah 1967) is the sum of all nil ideals.

Proof. By Theorem 3.7, $Q(N)=P_{0}(N)$. By Theorem 3.4 of Ramakotaiah (1967), $J_{-1}(N)$ is a nil ideal and hence $J_{-1}(N) \subseteq Q(N)=P_{0}(N)$. But we have $P_{0}(N) \subseteq J_{-1}(N)$. Therefore $J_{-1}(N)=P_{0}(N)$.

If an IFP near-ring $N$ is a ring we can say something about $N \backslash K$ for each maximal sub-semi-group $K$ in $N^{*}$. In the remaining part of this section we assume that $N$ is an IFP near-ring which is a ring. We remark that for each maximal sub-semigroup $K$ in $N^{*}, N \backslash K$ is precisely the set of elements $y \in N$ such that for some $x \in K, x y$ is nilpotent.

THEOREM 3.10. If $N$ is an IFP ring, then for each maximal sub-semi-group $K$ in $N^{*}$, $N \backslash K$ is an ideal of $N$.

PROOF. Let $y_{1}, y_{2} \in N \backslash K$. Then there exist $m_{1}, m_{2} \in K$ such that $m_{1} y_{1}$ and $m_{2} y_{2}$ are nilpotent. Therefore there exist integers $n_{1}$ and $n_{2}$ such that

$$
\left(m_{1} y_{1}\right)^{n_{1}}=\left(m_{2} y_{2}\right)^{n_{2}}=0 \text {. }
$$


Inserting $m_{2}$ 's in $\left(m_{1} y_{1}\right)^{n_{1}}=0$ and $m_{1}$ 's in $\left(m_{2} y_{2}\right)^{n_{2}}=0$ suitably, it can be shown that $\left(m_{1} m_{2} y_{1}\right)^{n_{1}}=\left(m_{1} m_{2} y_{2}\right)^{n_{2}}=0$. Let $n$ be an integer greater than or equal to $n_{1}+n_{2}$. A typical term in the expansion of $\left(m_{1} m_{2} y_{1}-m_{1} m_{2} y_{2}\right)^{n}$ is of the form $l\left(m_{1} m_{2} y_{1}\right)^{i_{1}} \ldots\left(m_{1} m_{2} y_{2}\right)^{i_{k}}$ where $l$ is an integer and $i_{1}+i_{2}+\ldots+i_{k}=n$. In this product either the sum of the powers of $m_{1} m_{2} y_{1}$ is greater than or equal to $n_{1}$ or the sum of the powers of $m_{1} m_{2} y_{2}$ is greater than or equal to $n_{2}$. In the former case, inserting the terms $m_{1} m_{2} y_{1}$ and $m_{1} m_{2} y_{2}$ suitably in $\left(m_{1} m_{2} y_{1}\right)^{n_{1}}=0$ we can show that the above typical term is zero. Similarly, the second case can be disposed of. Therefore $m_{1} m_{2}\left(y_{1}-y_{2}\right)$ is nilpotent and hence $y_{1}-y_{2} \in N \backslash K$, otherwise $K$ contains zero. For any $y \in N \backslash K$ and $n \in N$, it is easy to verify that $n y$ and $y n$ belong to $N \backslash K$. Therefore $N \backslash K$ is an ideal.

COROLLARY 3.11. If $N$ is an IFP ring, then for each maximal sub-semi group $K$ in $N^{*}, N \backslash K$ is a minimal prime ideal of type 1 .

Proof. Since $K$ is a maximal sub-semi-group in $N^{*}, N \backslash K$ is a minimal prime ideal of type 2. Therefore $N \backslash K$ is a prime ideal of type 1 . Let $P$ be any prime ideal of type 1 contained in $N \backslash K$. Let $y \in N \backslash K$. There exists $x \in K$ such that $(x y)^{l}=0$ for some positive integer $l$. Now for all $n \in N,(x n y)^{l}=0$. Since $P$ contains all nilpotent elements (by Theorem 3.5); $x n y \in P$ for all $n \in N$. Therefore $x N y \subseteq P$. Since $P$ is a prime ideal of type 1 and since $x \notin P, y \in P$. Therefore $N \backslash K=P$ and hence $N \backslash K$ is a minimal prime ideal of type 1 .

COROLLARY 3.12. In an IFP ring $N$, an ideal $P$ is a minimal prime ideal of type 2, if and only if $P=N \backslash K$ for some maximal sub-semi-group $K$ in $N^{*}$.

Proof. If $P=N \backslash K$ for some maximal sub-semi-group $K$ in $N^{*}$, then $P$ is a minimal prime ideal of type 2 . Conversely suppose $P$ is a minimal prime ideal of type 2. Put $K=N \backslash P$. Then $K$ is a sub-semi-group in $N^{*}$ and hence $K$ can be extended to a maximal semi-group-sub $K^{\prime}$ in $N^{*}$. Now $N \backslash K^{\prime}$ is a prime ideal of type 2 and $N / K^{\prime} \subseteq P$. Since $P$ is minimal, $P=N \backslash K^{\prime}$. Therefore $K=K^{\prime}$ and hence $P=N \backslash K$. This completes the proof of the theorem.

COROLLARY 3.13. If $N$ is an IFP ring then $Q(N)$ is the intersection of all minimal prime ideals of type 2.

The proof is easy and will be omitted.

Finally, we make the following characterization. 
THEOREM 3.14. If $N$ is an IFP ring, then the following statements are equivalent.

(1) $Q(N)$ is the intersection of all minimal prime ideals of type 2 .

(2) $Q(N)$ is the intersection of all prime ideals of type 1 .

(3) $Q(N)$ is the largest nil ideal of $N$.

Proof. (1) is the Corollary 3.13. (2) follows from Theorem 3.5. The proof of (3) follows from Theorem 2.4 and Corollary 3.8 .

\section{References}

H. E. Bell (1970), 'Near-rings in which each element is a power of itself', Bull. Austral. Math. Soc. 2, 363-368.

H. E. Bell (1971), 'Certain near-rings are rings', J. London Math. Soc. II, Ser. 4, 264-270.

S. Ligh (1970), 'On regular near-rings', Math. Japan. 15, 7-13.

S. Ligh (1970), 'On the commutativity of near-rings, I', Kyungpook Math. J. 10, 105-106.

S. Ligh (1971), 'On the commutativity of near-rings, II', Kyungpook Math. J. 11, 159-163.

S. Ligh (1972), 'On the commutativity of near-rings, III', Bull. Austral. Math. Soc. 6, 459-464.

G. Pilz (1977), Near-rings (North-Holland, Amsterdam).

D. Ramakotaiah (1967), 'Radicals for near-rings', Math. Z. 97, 45-46.

\section{Department of Mathematics}

Nagarjuna University

Nagarjunanagar 522510

Guntur (A.P) India 